\title{
HOMOLOGY OF DELETED PRODUCTS IN DIMENSION ONE
}

\author{
ARTHUR H. COPELAND, JR. ${ }^{1}$
}

If $X$ is a topological space, then the deleted product space of $X$ is the difference $R X=X \times X-\Delta X$, where $\Delta X$ is the diagonal subspace of the topological product $X \times X$. An embedding $f: X \rightarrow Y$ induces a map $R f: R X \rightarrow R Y$, and isotopic embeddings induce homotopic (in fact, isotopic) maps. Thus $X \rightarrow H_{k}(R X)$ is an isotopy functor when $H_{k}()$ is the $k$ th singular homology functor with integral coefficients [1]. The first section of this note shows that these isotopy functors pick out the isotopy equivalences between 1-dimensional finitely triangulable spaces. The second section presents a formula for computing $H_{k}(R X)$ when $X$ is a 1-dimensional finitely triangulable space.

\section{Isotopy equivalences.}

Theorem. If $X$ and $Y$ are finitely triangulable 1-dimensional spaces and if $f: X \rightarrow Y$ is an embedding, then

$$
R f_{*}: H_{k}(R X) \rightarrow H_{k}(R Y)
$$

is an isomorphism for all $k$ if and only if $f$ is an isotopy equivalence.

Proof. S.-T. Hu has shown that if $f$ is an isotopy equivalence, then $R f_{*}$ is an isomorphism in all dimensions [1]. then

Suppose $R f_{*}$ is an isomorphism. If $X$ has components $X_{1}, \cdots, X_{n}$,

$$
R X=\bigcup_{i=1}^{n} R X_{i} \cup \underset{i \neq j}{\bigcup} X_{i} \times X_{j}
$$

The summands in this union are mutually disjoint and are closed in $R X$. Thus if $f$ induces an isomorphism of the 0 -dimensional homology groups of the deleted products, then $f$ must induce a one-to-one correspondence between the components of $X$ and those of $Y$. It follows that $f$ induces isomorphisms between the homology groups of the deleted products of corresponding components. It suffices, then, to prove the theorem when $X$ and $Y$ are both connected.

The embedding $f: X \rightarrow Y$ may be regarded as an inclusion map, and the two spaces may be given triangulations which are consistent with

Received by the editors September 21, 1964.

1 This research was supported by NSF Grant G24154. 
each other. Next, factor the inclusion map $f: X=X_{0} \rightarrow X_{1} \rightarrow \cdots$ $\rightarrow X_{m}=Y$ in such a way that $X_{i}$ is formed from $X_{i-1}$ by the addition of a single 1-simplex $\sigma$. Take the triangulations sufficiently fine that these additions are of one of the following two types.

(a) Addition at both ends: $\sigma \cap X_{i-1}$ consists of the two endpoints of $\sigma$, and each has order 2 in $X_{i}$.

(b) Addition at one end: $\sigma \cap X_{i-1}$ is one of the two endpoints of $\sigma$ and is a vertex of order $s$ in $X_{i}$.

The order of a vertex is the number of 1-simplexes which meet it. C. W. Patty [2] shows that with an addition at both ends, the inclusion map induces a homomorphism $H_{1}\left(R X_{i-1}\right) \rightarrow H_{1}\left(R X_{i}\right)$ which is never an epimorphism, and that with an addition at one end, the induced homomorphism is an epimorphism only when the vertex has order $s=2$ in $X_{i}$. Thus if $R f_{*}$ is an isomorphism, each inclusion must be of the second type and have $s=2$. But such an embedding is an isotopy equivalence, whence $f$ is, also.

Note that the proof of necessity uses only the fact that $R f_{*}$ is an epimorphism in dimensions $k=0,1$.

It is not true that $X$ and $Y$ must have the same isotopy type whenever $R X$ and $R Y$ have isomorphic homology. The next section provides a wealth of counterexamples.

2. Betti numbers of deleted products. It is an immediate corollary to Patty's work that the homology groups $H_{k}(R X)$ are free abelian when $X$ is a 1-dimensional finitely triangulable space. Thus in order to describe these groups, it suffices to describe the $k$-dimensional Betti numbers $\beta_{k}$ of $R X$ for each $k$. Note that these are zero for $k>2$.

The 0-dimensional Betti number $\beta_{0}$. If $X$ consists of a single point, then $R X$ is empty. If $X$ is an arc, then $R X$ has two components. If $X$ is any other connected space, then $R X$ is connected. Thus if $X$ has $n$ components, if $p$ of these are isolated points and if $q$ are arcs, then it follows from formula (1) of $\$ 1$ that

$$
\beta_{0}=n^{2}-p+q \text {. }
$$

The 2-dimensional Betti number $\beta_{2}$. The group $H_{2}(X \times X)$ is generated by the 2-dimensional cycles of the tori $c \times c^{\prime} \subset X \times X$, where $c$ and $c^{\prime}$ are simple closed curves in $X$. The inclusion map induces a monomorphism $H_{2}(R X) \rightarrow H_{2}(X \times X)$, and the image is generated by the cycles $c \times c^{\prime}$ with $c$ and $c^{\prime}$ disjoint simple closed curves. The rank of this image is $\beta_{2}$.

The 1-dimensional Betti number $\beta_{1}$. Let $X$ be given a fixed triangulation. W.-T. Wu [3] has shown that the inclusion map of

$$
J X=\bigcup\{x \times y: x, y \text { simplexes of } X \text { and } x \cap y \text { empty }\}
$$


in $R X$ is a homotopy equivalence. The cells $x \times y$ form a cellular decomposition of $J X$. For $k=0,1,2$, let $c_{k}$ and $c_{k}^{\prime}$ be the number of $k$ dimensional cells in $X$ and $J X$, respectively. If $x$ is a simplex of $X$, let $a(x)$ be the number of 1 -simplexes in $X$ which meet $x ; x$ itself is numbered among these in case it is a 1 -simplex. If $u_{i}\left(i=1, \cdots, c_{0}\right)$ are the 0 -simplexes of $X$ and if $v_{i}\left(i=1, \cdots, c_{1}\right)$ are the 1 -simplexes, then

$$
\begin{aligned}
& c_{0}^{\prime}=c_{0}^{2}-c_{0}, \\
& c_{1}^{\prime}=2 \sum_{i=1}^{c_{0}}\left(c_{1}-a\left(u_{i}\right)\right)=2 c_{0} c_{1}-4 c_{1}, \\
& c_{2}^{\prime}=\sum_{i=1}^{c_{1}}\left(c_{1}-a\left(v_{i}\right)\right)=c_{1}^{2}-\sum_{i=1}^{c_{1}} a\left(v_{i}\right) .
\end{aligned}
$$

It follows at once from these three equations and the Euler-Poincaré formula that

$$
\beta_{1}=\beta_{0}+\beta_{2}-4 c_{1}+c_{0}-\left(c_{0}-c_{1}\right)^{2}+\sum_{i=1}^{c_{1}} a\left(v_{i}\right) .
$$

\section{REFERENCES}

1. S.-T. Hu, Isotopy invariants of topological spaces, Proc. Roy. Soc. London. Ser. A 255 (1960), 331-366.

2. C. W. Patty, The fundamental group of certain deleted product spaces, Trans. Amer. Math. Soc. 105 (1962), 314-321.

3. W.-T. Wu, On the realization of complexes in euclidean spaces. I, Acta Math. Sinica 5 (1955), 505-552.

\section{NORTHWESTERN UNIVERSITY}

